\title{
3C 33: another case of photoionized soft X-ray emission in radio galaxies
}

\author{
E. Torresi ${ }^{1,2}$, P. Grandi ${ }^{1}$, M. Guainazzi ${ }^{3}$, G. G. C. Palumbo ${ }^{2}$, G. Ponti ${ }^{4}$, and S. Bianchi ${ }^{5}$ \\ 1 Istituto di Astrofisica Spaziale e Fisica Cosmica-Bologna, INAF, via Gobetti 101, 40129 Bologna, Italy \\ e-mail: torresi@iasfbo.inaf.it \\ 2 Dipartimento di Astronomia, Università di Bologna, via Ranzani 1, 40127 Bologna, Italy \\ 3 European Space Astronomy Center of ESA, Apartado 50727, 28080 Madrid, Spain \\ 4 APC, Université Paris 7 Denis Diderot, 75205 Paris Cedex 13, France \\ 5 Dipartimento di Fisica, Università degli Studi Roma Tre, via della Vasca Navale 84, 00146 Roma, Italy
}

Received 9 September 2008 / Accepted 14 January 2009

\section{ABSTRACT}

\begin{abstract}
We investigate the X-ray spectral properties of 3C 33 by analyzing all the observations available in the Chandra and XMM-Newton archives. In this paper we present a complete X-ray analysis of the nuclear emission of this narrow-line radio galaxy. The broad-band spectrum of 3C 33 is complex. Its hard part resembles that of Seyfert 2 galaxies, which have a heavily obscured nuclear continuum $\left(N_{\mathrm{H}} \sim 10^{23} \mathrm{~cm}^{-2}\right)$ and a prominent $\mathrm{Fe} \mathrm{K} \alpha$ line. This represents the nuclear radiation observed directly in transmission through a cold circumnuclear gas. On the other hand an unabsorbed continuum plus emission lines seem to reproduce the soft part of the spectrum $(0.5-2 \mathrm{keV})$ well, suggesting that the jet does not contribute significantly to the X-ray emission. We discuss the possible collisional or photoionized origin of the gas that emits the soft X-ray lines. Our results, supported by those from optical spectroscopy, favor the photoionization scenario.
\end{abstract}

Key words. galaxies: active - X-rays: galaxies - X-rays: individuals: 3C 33

\section{Introduction}

With the advent of high-resolution grating spectrometers, X-ray spectroscopy has become the most powerful tool for investigating the ionized component of the gaseous AGN environment. The detection of emission lines in Seyfert 2 galaxies has revealed gas photoionized by the nuclear engine (Sako et al. 2000a,b; Kinkhabwala et al. 2002; Bianchi et al. 2005; Pounds \& Vaughan 2006; Guainazzi \& Bianchi 2007).

Spatially resolved studies realized with the Chandra telescope for at least the three brightest AGNs, NGC 1068 (Kinkhabwala et al. 2002; Brinkman et al. 2002; Ogle et al. 2003), Circinus (Sambruna et al. 2001b), and Mkn 3 (Sako et al. 2000a,b; Bianchi et al. 2005b; Pounds \& Page 2005), have revealed that the soft $\mathrm{X}$-ray emission originates in regions extended on hundreds of $\mathrm{pc}$, and the dimension and morphology of this emission closely resembles that of the Narrow Line Region (NLR) as mapped by the [OIII] $\lambda 5007$ emission line (see e.g., Bianchi et al. 2006).

Systematic studies of large samples of FRII Narrow Line Radio Galaxies (NLRG) found that the High Excitation Radio Galaxies (HEG; Jackson \& Rawlings 1997) have a heavily obscured continuum (intrinsic column density $\sim 10^{23-24} \mathrm{~cm}^{-2}$ ) related to the bright accretion disk obscured by an edge-on oriented thick torus, and a soft unabsorbed tail (Belsole et al. 2006; Hardcastle et al. 2006; Evans et al. 2006).

Soft X-ray emission above the extrapolation of the obscured component is often detected. The nature of the soft X-ray emission in radio galaxies is still a matter of debate. Radioloud AGNs are potentially more complex than their radio-quiet counterparts, because the jet could also contribute to the X-ray emission.

It was suggested that the soft excess is related to the jet emerging from the edge of the dusty torus (Hardcastle \& Worrall 1999; Belsole et al. 2006). Recently the discovery of emission lines produced by the photoionized gas in the soft X-ray spectrum of 3C 445 (Grandi et al. 2007; Sambruna et al. 2007) and 3C 234 (Piconcelli et al. 2008) provided an alternative way of interpreting the soft excess.

$3 \mathrm{C} 33$ is the ideal target for investigating the nature of the soft excess. It is a genuine type II radio galaxy, optically classified as High Excitation Radio Galaxy (HEG) and one of closest HEG in the 3CRR catalogue (Laing et al. 1983) located at $z=0.0597$ (Popescu et al. 1996).

Its radio morphology is typical of FRII radio galaxies $\left(L_{178}=\right.$ $7.5 \times 10^{25} \mathrm{~W} \mathrm{~Hz}^{-1} \mathrm{sr}^{-1}$ ), exhibiting symmetric lobes with hot spots on kpc scale (Leahy \& Perley 1991), and two symmetric jets in the VLBI image (Giovannini et al. 2005). The southern jet is stronger by a factor 2 at 5 mas. Expressing the jet/counterjet ratio $(J)$ in terms of the bulk velocity of the jet $(\beta)$ and the angle between the jet and the line of sight $(\theta)^{1}$, an inclination angle of $75^{\circ}-80^{\circ}$ can be deduced. Its spatial orientation is then favorable for revealing soft X-ray emission lines, because a strong de-amplification of the jet radiation is expected.

Moreover $3 \mathrm{C} 33$ is very luminous in the [OIII] band $\left(L_{[\mathrm{OIII}]} \sim\right.$ $1.09 \times 10^{42} \mathrm{erg} \mathrm{s}^{-1}$ ) since it exhibits the highest [OIII] flux of all nearby NLRGs $(z<0.3)$ in the 3CRR sample. Previous nuclear observations of 3C 33 in the X-ray band date to those of the EINSTEIN observatory (Fabbiano et al. 1984) and ROSAT

\footnotetext{
${ }^{1} \beta \cos \theta=\frac{J^{1 / p}-1}{J^{1 / p}+1}, p=2 \gamma=2,10$. From Urry \& Padovani (1995).
} 
(Hardcastle et al. 1998; Hardcastle \& Worrall 1999) X-ray telescopes. EINSTEIN only provided an upper limit to the 0.5-3 keV luminosity, while ROSAT/HRI could detect the source in the soft X-ray band, although the HRI instrument onboard was not able to resolve the source.

Evans et al. (2006) analyzed the XMM-Newton EPIC PN data of 3C 33 showing for the first time the Seyfert 2 nature of its hard component. They modeled the soft component with an unabsorbed power law.

Taking advantage of the unprecedented imaging of Chandra, Kraft et al. (2007) performed a detailed study of 3C 33, resolving the north and south hot-spot regions. They also studied the nuclear component with a basic model consisting of an absorbed power law plus a $\mathrm{Fe} \mathrm{K} \alpha$ line and a thermal component. Large residuals between 1 and $3 \mathrm{keV}$ could be explained by assuming the presence of either a reflection component or a less absorbed power law, which they interpreted as jet emission on parsec scale. Kraft et al. (2007) used thermal gas to model the soft $\mathrm{X}$-ray emission on the basis of the Chandra imaging that reveals in the soft band $(0.3-0.7 \mathrm{keV})$ a clearly extended structure around the unresolved hard nucleus. A similar elongation was observed in the WFPC1 and WFPC2 Hubble Space Telescope imaging, and was interpreted by the authors as a tracer of stellar distribution.

In this work, we reanalyzed all the Chandra and XMM-Newton observations of the radio galaxy 3C 33 available in the public archives, and propose an alternative interpretation of the nature of the soft X-ray emission of 3C 33.

\section{Observations and data reduction}

Chandra ACIS-S observed 3C 33 twice $(\sim 20 \mathrm{ks}$ on 2005 November 8 (ObsID 6190) and 2005 November 12 (ObsID 7200)). Spectra and instrument responses were generated using the Chandra Interactive Analysis of Observations (CIAO) 3.4 and the Chandra Calibration Database (CALDB) 3.4.3. The source spectrum was extracted from a circular region of radius $1.5^{\prime \prime}$ centered on the peak of the X-ray emission, while background counts were extracted from a 4 " radius circle within the same $\mathrm{CCD}$, but outside the extended emission of the source. The Chandra count rate from the nucleus in both observations was $\sim 0.06 \mathrm{cts} \mathrm{s}^{-1}$, implying a negligible pileup effect.

In the hard band $(2-8 \mathrm{keV})$, the X-ray morphology of the 3C 33 nucleus is point-like. An extended structure around the nucleus becomes evident in the soft band $(0.5-2 \mathrm{keV})$, looking approximately elliptical, with a major axis of $\sim 5^{\prime \prime}$, elongated in the northeast-southwest (NE-SW) axis, in agreement with the analysis of Kraft et al. (2007).

XMM-Newton observed 3C 33 twice, on 2004 January 4 and January 21 (ObsID 0203280101; 0203280301). We checked both observations but analyzed only the second pointing ( $\sim 9 \mathrm{ks})$ because the first was affected by high flaring activity, which prevented us from obtaining useful information from the RGS analysis.

The EPIC cameras were operated in "prime full window" mode. The data were reduced using the SAS (v. 7.1) with standard procedures.

The light curve over $10 \mathrm{keV}$ was extracted to check high background periods. We excluded the time intervals characterized by a count rate higher than $1.0 \mathrm{cts} / \mathrm{s}$ in the EPIC-PN, and $0.35 \mathrm{cts} / \mathrm{s}$ in the EPIC-MOS. After this data cleaning, we obtained a net exposure of $6.3 \mathrm{ks}$ for the PN and $8.5 \mathrm{ks}$ for the two MOS cameras.
The source and the background spectra were extracted from circular regions of $35^{\prime \prime}$ radius. The background was taken from the same CCD of 3C 33, but within a region that was not contaminated by the source. The source counts were $\sim 1500$ and $\sim 1000$ in the PN and the two combined MOS, respectively. The response matrices were created using the SAS commands RMFGEN and ARFGEN.

Events outside the $0.5-10 \mathrm{keV}$ band were discarded in both the PN and MOS spectra (Kirsch 2006).

All spectral fits presented in this paper include absorption due to a line-of-sight Galactic column density of $N_{\mathrm{H}}=3.06 \times$ $10^{20} \mathrm{~cm}^{-2}$ (Kalberla et al. 2005).

Errors are quoted at the $90 \%$ confidence level $\left(\Delta \chi^{2}=2.7\right)$ for one interesting parameter.

The cosmological parameters used throughout the paper are $H_{0}=70 \mathrm{~km}^{-1} \mathrm{~s}^{-1} \mathrm{Mpc}^{-1}, \Omega_{\mathrm{m}}=0.3, \Omega_{\Lambda}=0.7$ (Spergel et al. 2007). For 3C 33, $z=0.0597$ corresponds to a luminosity distance of $263.9 \mathrm{Mpc}$, and $1^{\prime \prime}$ corresponds to a physical scale of $1.14 \mathrm{kpc}$.

The spectral analysis obtained by both telescopes was performed using the XSPEC 11.3 package.

\section{Spectral analysis}

We initiated our study using Chandra data. The two observations were analyzed simultaneously using spectra extracted from $1.5^{\prime \prime}$ regions around the nucleus. We also considered a larger extraction radius for the nuclear source $\left(r=4.5^{\prime \prime}\right)$ to include the extended soft X-ray emission. As expected, the statistical quality of the spectrum decreased due to the higher background. Few source counts were detected in the $(0.5-2) \mathrm{keV}$ band from an elliptical region of 5.5" semi-major axis and $4^{\prime \prime}$ semi-minor axis (excluding the nucleus), less than $3 \%$ of the total counts in the $1.5^{\prime \prime}$ region. In physical terms most of the soft X-ray radiation comes from $\sim 2 \mathrm{kpc}$ around the source. We then considered the smaller nuclear region in order to maximize the spectrum signalto-noise ratio $(\mathrm{S} / \mathrm{N})$.

\subsection{Hard X-ray spectrum}

The broad-band spectrum appeared quite complex. We initially decided to fit only the energy range $3-10 \mathrm{keV}$ in order to focus on the hard X-ray continuum and to exclude the soft emission. At first, we applied a photoelectrically absorbed power-law plus a Gaussian line profile. The restricted energy band and the limited statistics did not allow us to constrain very well the spectral index (the column density and the spectral index being strongly correlated), so we decided to fix $\Gamma=1.7$, following Kraft et al. (2007). This choice was confirmed to be the right one by the $X M M$-Newton analysis (see below). Since the $\chi^{2}=102(80)$ was not completely satisfactory, we tested for the presence of a reflection component, which is often required in obscured AGNs (see for example, Mkn3, Pounds \& Page 2005; and 3C 445, Grandi et al. 2007; Sambruna et al. 2007). When a (PEXRAV) (Magdziarz \& Zdziarski 1995) model was applied the value of $\chi^{2}$ decreased to 82 for 79 d.o.f., providing a more accurate representation of the data $\left(P_{F} 99.99 \%\right)$. The reflection $(R=\Omega / 2 \pi)$ was allowed to vary, while the high-energy cut-off of the incident power law was set at $160 \mathrm{keV}$ (Grandi et al. 2006), and the elemental abundances were assumed to be of solar value. We repeated this analysis allowing $\Gamma$ to vary freely. Also in this case, the addition of a reflection component improves the fit, even if the strong correlation between the reflection component, $\Gamma$, and 

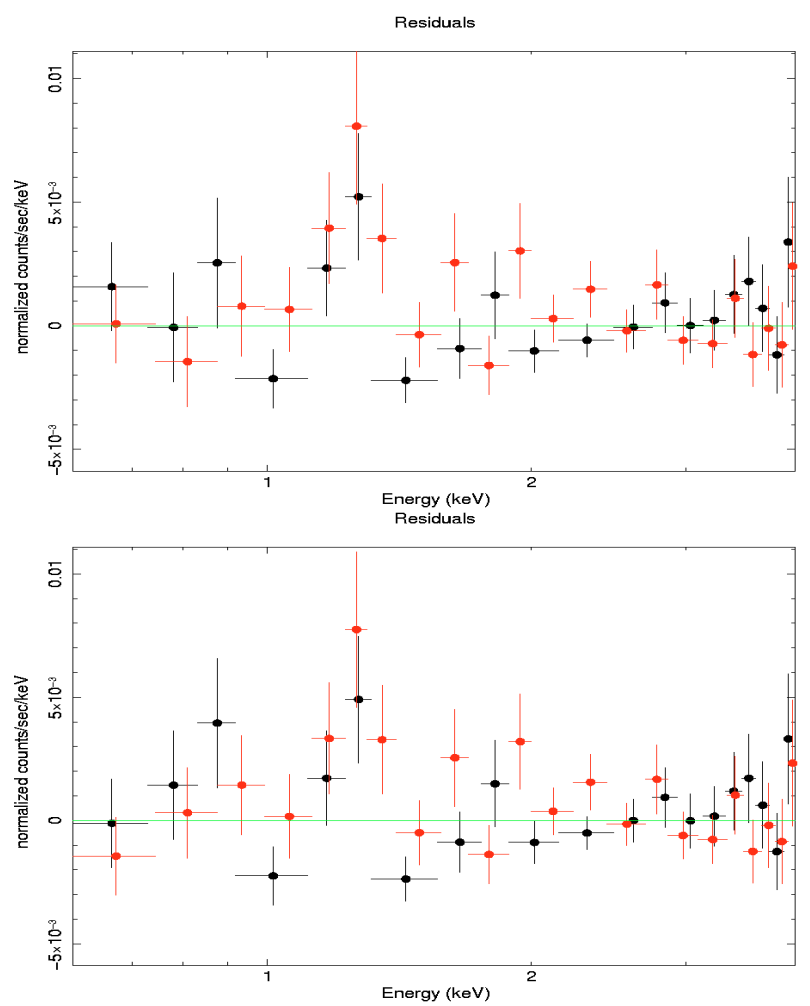

Fig. 1. Residuals corresponding to the two different soft excess models: up two mekal and down a scattered power-law.

$N_{\mathrm{H}}$ prevented us from being able to constrain these three variables accurately.

The FeK $\alpha$ emission line is unresolved. The rest-frame energy is $E_{\mathrm{K} \alpha}=6.38_{-0.06}^{+0.05} \mathrm{keV}$, the upper limit of the intrinsic width is $\sigma<180 \mathrm{eV}$ and the equivalent width is $E W_{\mathrm{K} \alpha}=172_{-76}^{+84} \mathrm{eV}$.

\subsection{Soft $X$-ray spectrum}

Once a good fit of the hard component had been obtained, we extended our study to the soft part $(0.5-3 \mathrm{keV})$ of the spectrum. The simple extrapolation of the hard model introduced a strong soft component. In the literature, at least three different interpretations of the soft excess in obscured radio-loud AGNs are proposed: i) thermal radiation from collisional gas (Kraft et al. 2007; Evans et al. 2006); ii) non-thermal emission from a jet (Belsole et al. 2006); iii) nuclear scattered radiation plus emission lines produced by circumnuclear photoionized gas (Bianchi \& Guainazzi 2007; Grandi et al. 2007; Sambruna et al. 2007; Piconcelli et al. 2008). All of these three possibilities were tested.

At first, we tried to reproduce the soft excess with a thermal model (mekal) absorbed by the Galactic column density. This implicitly assumed that the soft excess is produced by a collisional plasma (with solar abundances). The gas temperature required by the fit was $k T \sim 0.7$, and the model partially reproduced the soft excess, $\chi^{2}$ (d.o.f.) $=138(104)$, but the inspection of the residuals still showed excesses below $2 \mathrm{keV}$. A second mekal was added to verify whether a multi-temperature gas was present, but the second temperature shifted to an improbable value higher than $10 \mathrm{keV}$, and excesses were still present in the soft X-ray band (see Fig. 1 upper panel). Note that if the abundances are free to vary, they become extremely low, resembling a power-law. After replacing the "second" thermal
Table 1. Chandra ACIS best-fit parameters.

\begin{tabular}{|c|c|}
\hline$\Gamma$ & $1.7^{*}$ \\
\hline$R$ & $2.18_{-0.60}^{+0.48}$ \\
\hline$N_{\mathrm{G}}$ & $\left(3.06 \times 10^{20}\right) \mathrm{cm}^{-2}$ \\
\hline$N_{\mathrm{H}}$ & $\left(5.28_{-0.42}^{+1.04}\right) \times 10^{23} \mathrm{~cm}^{-2}$ \\
\hline$n$ & $\left(1.83_{-0.33}^{+0.51}\right) \times 10^{-3}$ \\
\hline$n_{\text {unobscured }}$ & $\left(4.48_{-2.11}^{+3.43}\right) \times 10^{-6}$ \\
\hline$E_{\mathrm{Fe}}(\mathrm{keV})$ & $6.38_{-0.06}^{+0.05}$ \\
\hline$F_{\mathrm{Fe}}$ & $\left(2.49_{-1.11}^{+1.21}\right) \times 10^{-5}$ \\
\hline$E W(\mathrm{eV})$ & $172_{-76}^{+84}$ \\
\hline$\chi^{2}$ (d.o.f.) & $103(99)$ \\
\hline \multicolumn{2}{|c|}{ Unabsorbed luminosity and absorbed flux } \\
\hline$L_{2-10 \mathrm{keV}}\left(\mathrm{erg} \mathrm{s}^{-1}\right)$ & $7.31 \times 10^{43}$ \\
\hline $\operatorname{Flux}_{2-10 \mathrm{keV}}\left(\mathrm{erg} \mathrm{s}^{-1} \mathrm{~cm}^{-2}\right)$ & $2.67 \times 10^{-12}$ \\
\hline
\end{tabular}

* Value fixed.

Normalizations $n$ are expressed in $\mathrm{cm}^{-2} \mathrm{~s}^{-1} \mathrm{keV}^{-1}$; the normalization of the iron line is expressed in photons $\mathrm{cm}^{-2} \mathrm{~s}^{-1}$.

Table 2. Emission lines detected in the Chandra ACIS spectrum.

\begin{tabular}{cccc}
\hline \hline Energy & Intensity & $P_{\mathrm{F}}$ & Tentative IDs \\
\hline $0.76_{-0.13}^{+0.02}$ & $2.93_{-1.02}^{+1.16}$ & $>98.9 \%$ & OVII RRC, Fe XVII 3s-2p \\
$0.93_{-0.02}^{+0.04}$ & $2.51_{-1.20}^{+0.85}$ & $>99 \%$ & Ne IX rif \\
$1.30_{-0.02}^{+0.04}$ & $1.41 \pm 0.46$ & $>99.97 \%$ & Mg XI He $\alpha$
\end{tabular}

$E_{\mathrm{c}}$ is the centroid line energy $(\mathrm{keV})$ in the source rest frame; $F$ is the line flux in units of $10^{-6}$ photons $\mathrm{cm}^{-2} \mathrm{~s}^{-1} ; P_{\mathrm{F}}$ is the significance of the fit improvement after including the Gaussian line in the model, based on the F-test.

emission model with a simple power-law (where $\Gamma_{\mathrm{s}}$ is free to vary), that represents a non-thermal emission, the $\chi^{2}$ improved $\chi^{2}$ (d.o.f.) $=124(103)$, and the thermal emission was no longer required (the mekal model is consistent with zero). Although this was the best fit obtained so far, the model could not reproduce the soft X-ray data completely (Fig. 1, lower panel).

Finally the third hypothesis was verified. A soft excess was fitted with a power law plus emission lines, as expected in case of circumnuclear photoionized gas. The soft power-law slope, $\left(\Gamma_{\mathrm{s}}\right)$, was linked to the hard one, $\left(\Gamma_{\mathrm{h}}\right)$, in order to represent the scattered nuclear continnum. The fit (see Fig. 2 upper panel) was very satisfactory $\chi^{2}$ (d.o.f.) $=103(99)$. A summary of the best-fit model parameters for the hard X-ray spectra is given in Table 1, while the detected lines and relative significance levels are listed in Table 2.

To obtain independent confirmation of this result, we explored the XMM-Newton EPIC spectra. The PN and MOS results were reassuring. All the EPIC spectra (see Fig. 2, lower panel) were fitted well with the same best-fit model as found for the Chandra continuum. Interesting enough, the PN data even required soft lines at the same energies measured by Chandra.

The PN best-fit model parameters are listed in Table 3. The relative $\chi^{2}$ is 37.5 for 37 degrees of freedom. 

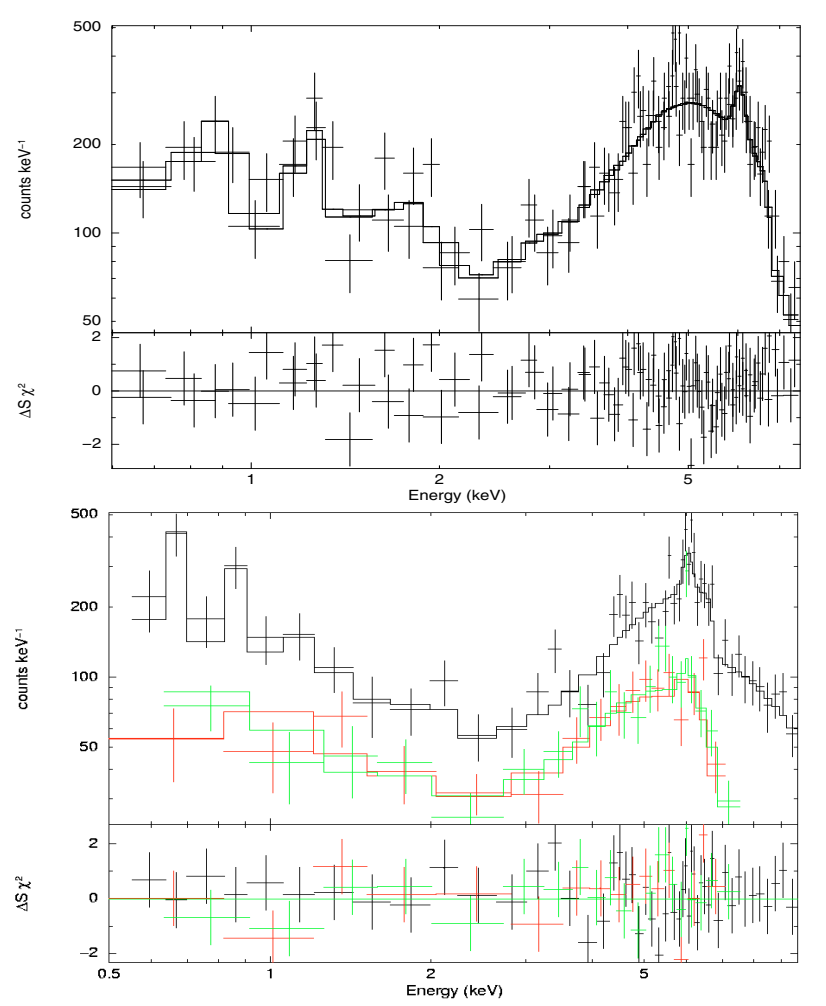

Fig. 2. Chandra spectra (upper panel) and XMM spectra (lower panel) of 3C 33. PN, MOS 1 and MOS 2 spectra are represented with black, red and green crosses, respectively. Residuals are in terms of standard deviations of the observed data from the model.

\section{Results}

Our analysis displays typical features produced by a cold circumnuclear gas: an unresolved $(\sigma<180 \mathrm{eV})$ narrow iron line $(E W \simeq 200 \mathrm{eV})$, and a reflection component $(R \simeq 2)$ (Risaliti 2002), highlighting the Seyfert 2 nature of the hard spectrum of $3 \mathrm{C} 33$. Both these values are consistent with the Chandra results of Kraft et al. (2007) (their Model 1).

The reflection component was obtained by assuming an arbitrarily inclination angle of $\theta \simeq 60^{\circ}(\mathrm{PEXRAV}$ parameter $\cos \theta \simeq$ $0.45)$. However, even considering a larger inclination angle, as suggested by the radio data, the value of $R$ does not change significantly. This is unsurprising because of the limited statistics and the large number of parameters involved in the fit. Although the reflection component is slightly higher than that expected from a homogeneous dusty torus, it is not unusual and often observed in radio-quiet obscured objects. This is probably indicative of a more complex inhomogeneous and structured circumnuclear absorber (Risaliti et al. 2002).

The spectral similarity between Seyfert $2 \mathrm{~s}$ and obscured radio galaxies appears to persist at soft X-ray energies, where the best-fit requires emission lines superimposed on a weak continuum. The lines in Tables 2 and 4 are likely produced by $\mathrm{H}$ - and He-like ions of light elements such as oxygen and neon, often detected in obscured AGNs which is indicative of photoionized gas (Bianchi \& Guainazzi 2007; Grandi et al. 2007; Sambruna et al. 2007; Piconcelli et al. 2008). Although the moderate ACIS energy resolution does not allow us to draw strong conclusions, we emphasize that the centroids of the lines occurs where two strong features produced by photoionized gas are expected, i.e., the OVII recombination feature and the NeIX triplet lines. Incidentally, we note that the intrinsic nuclear luminosity
Table 3. XMM-Newton EPIC best-fit parameters.

\begin{tabular}{|c|c|}
\hline$\Gamma$ & $1.69_{-0.10}^{+0.18}$ \\
\hline$R$ & $2.7_{-0.60}^{+0.81}$ \\
\hline$N_{\mathrm{G}}$ & $\left(3.06 \times 10^{20}\right) \mathrm{cm}^{-2}$ \\
\hline$N_{\mathrm{H}}$ & $\left(7.14_{-1.21}^{+1.75}\right) \times 10^{23} \mathrm{~cm}^{-2}$ \\
\hline$n$ & $\left(1.83_{-0.31}^{+0.47}\right) \times 10^{-3}$ \\
\hline$n_{\text {unobscured }}$ & $\left(8.1_{-7.28}^{+4.08}\right) \times 10^{-6}$ \\
\hline$E_{\mathrm{Fe}}(\mathrm{keV})$ & $6.40_{-0.08}^{+0.13}$ \\
\hline$F_{\mathrm{Fe}}$ & $\left(3.65_{-2.04}^{+3.93}\right) \times 10^{-5}$ \\
\hline$E W(\mathrm{eV})$ & $206_{-115}^{+199}$ \\
\hline$\chi^{2}$ (d.o.f.) & 37.5(37) \\
\hline \multicolumn{2}{|c|}{ Unabsorbed luminosity and absorbed flux } \\
\hline$L_{2-10 \mathrm{keV}}\left(\mathrm{erg} \mathrm{s}^{-1}\right)$ & $7.53 \times 10^{43}$ \\
\hline Flux $_{2-10 \mathrm{keV}}\left(\mathrm{erg} \mathrm{s}^{-1} \mathrm{~cm}^{-2}\right)$ & $2.64 \times 10^{-12}$ \\
\hline
\end{tabular}

Normalizations $n$ are expressed in $\mathrm{cm}^{-2} \mathrm{~s}^{-1} \mathrm{keV}^{-1}$; the normalization of the iron line is expressed in photons $\mathrm{cm}^{-2} \mathrm{~s}^{-1}$.

Table 4. Emission lines detected in the XMM-Newton EPIC spectrum.

\begin{tabular}{lccc}
\hline \hline Energy & Intensity & $P_{\mathrm{F}}$ & Tentative IDs \\
\hline $0.71 \pm 0.03$ & $7.89_{-4.6}^{+2.8}$ & $>99.8 \%$ & OVII RRC, Fe XVII 3s-2p \\
$0.93_{-0.05}^{+0.03}$ & $5.23_{-4.8}^{+3.1}$ & $>99 \%$ & Ne IX rif \\
$1.26_{-0.17}^{+0.04}$ & $2.35 \pm 2.2$ & $>88 \%$ & Mg XI He $\alpha$ \\
\hline
\end{tabular}

$E_{\mathrm{c}}$ is the centroid line energy $(\mathrm{keV})$ in the source rest frame; $F$ is the line flux in units of $10^{-6}$ photons $\mathrm{cm}^{-2} \mathrm{~s}^{-1} ; P_{\mathrm{F}}$ is the significance of the fit improvement after including the Gaussian line in the model, based on the F-test.

of 3 C $33\left(L_{1-1000 \mathrm{ryd}} \sim 2 \times 10^{44} \mathrm{erg} \mathrm{s}^{-1}\right)$ is comparable to those observed in typical Seyfert $2 \mathrm{~s}$, for which the higher data quality has permitted detailed spectral studies of the soft X-ray diagnostics (see, for example, Pounds \& Page 2005; Sako et al. 2000a,b). Approximate computations indicate that the energy budget of the AGN is sufficient in producing a range of values of the ionization parameter $\left(\xi=\frac{L_{1-1000 \mathrm{ryd}}}{n_{\mathrm{e}} R^{2}}\right)$ consistent with the photoionized scenario (Kinkhabwala et al. 2002). If the soft X-ray emission is related to the Narrow Line Region (as in Seyfert 2s), assuming a minimum $\left(R_{\min } \sim 1 \mathrm{pc}\right)$ and a maximum $\left(R_{\max } \sim\right.$ $2 \mathrm{kpc}$, our nuclear extraction region) distance of the gas, a typical NLR electron density $n_{\mathrm{e}} \sim 10^{3} \mathrm{~cm}^{-3}$ implies a value of $\log \xi$ spanning between -2 and $4 \mathrm{erg} \mathrm{cm} \mathrm{s}^{-1}$.

\section{The origin of the soft X-ray emission}

\subsection{Imaging}

$\mathrm{X}$-ray spectroscopy provides evidence for the presence of soft $\mathrm{X}$-ray emission lines in the radio galaxy 3C 33.

Despite the limited spectral resolution of the CCDs, we could detect features that are plausibly emitted by a photoionized gas (see Tables 2 and 4). 


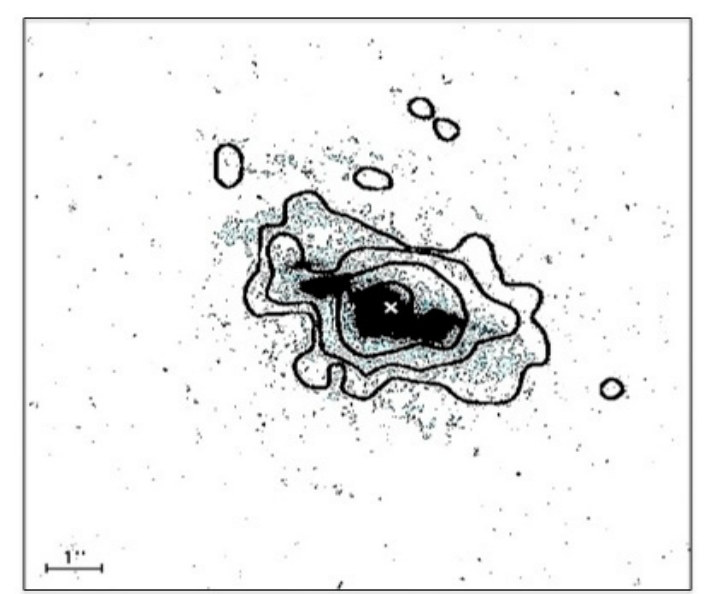

Fig. 3. Superposition of the Chandra soft X-ray $(<2 \mathrm{keV})$ contours on an HST image taken through a linear ramp filter at redshifted $[\mathrm{OIII}] \lambda 5007$. The sign " $\mathrm{x}$ " indicates the centre of the hard $\mathrm{X}$-ray source, north is up, east to the left. The X-ray image was smoothed with a Gaussian of $F W H M \sim 6$ pixels. The contours correspond to four logarithmic intervals in the range $1-60 \%$ of the peak flux.

The analogy between Seyfert 2 galaxies and Narrow Line Radio Galaxies is straightforward. Narrow Line Radio Galaxies have similar continua and environments to their radio-quiet counterparts. In Seyfert 2 galaxies, the coincidence in morphology and dimension of the soft X-ray and the [OIII] $\lambda 5007$ emission suggests that the Narrow Emission Line Region is the location of this gas. It is then natural to search for a similar X-ray and optical spatial correlation in 3C 33.

For this reason, the narrow-band HST ACS/WFC1 optical image, centred at redshifted [OIII] $\lambda 5007$ (filter FR551N), was retrieved from the Multi Mission Archive at STScI (MAST) and aligned with the Chandra image as described by Bianchi et al. (2006).

Figure 3 shows the Chandra soft X-ray contours $(<2 \mathrm{keV})$ superimposed on the HST [OIII] image. The coincidence in terms of extension and morphology between the two type of emissions is impressive.

\subsection{Further hints from optical spectroscopy}

Further support of the photoionization scenario comes from optical spectroscopy.

Several line diagnostic diagrams have been proposed to discriminate between collisionally ionized plasma (e.g., starburst emission) and photoionized plasma irradiated by a central engine (see for example Miller et al. 2003). One of these, based on the $[\mathrm{OIII}] / \mathrm{H} \beta$ and $[\mathrm{NII}] / \mathrm{H} \alpha$ ratios (Fig. 3 of Miller et al. 2003) could be used to ascertain the nature of the gas emission of $3 \mathrm{C} 33$. Using the nuclear reddening-corrected emissionline luminosities reported by Koski (1978), we estimated that $\log ([\mathrm{OIII}] / \mathrm{H} \beta) \sim 1.1$ and $\log ([\mathrm{NII}] / \mathrm{H} \alpha) \sim-0.17$. These values imply that $3 \mathrm{C} 33$ occupies a region of the diagram populated by the photoionized AGNs.

Baum et al. (1992) performed an accurate study of the extended $\mathrm{kpc}$ emission-line region (EELR), and measured a large value of $[\mathrm{OIII}] / \mathrm{H} \beta$ ratio, which they interpreted as being produced by photoionized gas with a high ionization parameter.

This result strengthens the early conclusion of Baum \& Heckman (1989), who demonstrated that the number of photons needed to photoionize the emission-line nebula of this source equals the number of ionizing photons coming from the central engine.

On the base of the kinematic classification of powerful radio galaxies proposed by Baum et al. (1992), 3C 33 also belongs to the rotator class. Rotators, characterized by a systematic gradient in the velocity of the emission-line gas, are nearly always associated with powerful radio sources $\left(L_{\text {radio }} \geq 10^{42} \mathrm{erg} \mathrm{s}^{-1}\right)$ having a luminous high-ionization nuclear emission-line region.

The kinematic study of the gas also shows that the gas rotation axis and the radio axis are aligned within $10^{\circ}$ (Baum et al. 1992). Moreover, the rotation axis of the gas is not aligned with the stellar rotation axis (Heckman et al. 1985), disfavoring the hypothesis of a collisional (starburst) gas emission.

\section{Conclusions}

An X-ray analysis of the Narrow Line Radio Galaxy 3C 33 has been presented using all the Chandra and XMM-Newton observations available in the public archives. Our study confirms the Seyfert 2 nature of this source. We agree with previous works that the broad-band spectrum is complex and largely dominated by strong obscuration $\left(N_{\mathrm{H}} \sim 10^{23} \mathrm{~cm}^{-2}\right)$.

A prominent $\mathrm{Fe} \mathrm{K} \alpha$ line at $6.4 \mathrm{keV}$ is also present. Its $E W$ of $\sim 200 \mathrm{eV}$ is in agreement with being produced by optically-thick circumnuclear matter.

We advocate photoionized gas as being responsible for the soft X-ray emission. The clues favoring this hypothesis are:

1. both Chandra and XMM-Newton soft X-ray spectra can be fitted more accurately with highly ionized lines and a unobscured scattered power-law, rather than with thermal components;

2. a rough estimate of the ionization parameter $\xi$, expected in the case that the emitting line gas is located in the NLR, is consistent with a photoionized scenario;

3. there is a remarkable spatial coincidence between the extended soft X-ray and the [OIII] $\lambda 5007$ emission;

Our X-ray findings are also consistent with pieces of evidence coming from other wavelengths such as:

4. the nuclear line ratios $[\mathrm{OIII}] / \mathrm{H} \beta$ and $[\mathrm{NII}] / \mathrm{H} \alpha$ place $3 \mathrm{C} 33$ in the right position of the line diagnostic plane of Miller et al. (2003), among the AGNs, far away from the region populated by starburst galaxies;

5. the extended kpc emission region has a line-rich optical spectrum with a large $[\mathrm{OIII}] / \mathrm{H} \beta$ ratio, typical of photoionized gas;

6. the estimated photons required to photoionize the emissionline nebula (in the optical band) are provided entirely by the nuclear engine;

7. the kinematical properties of the extended gas of $3 \mathrm{C} 33$, based on optical spectroscopy, are typical of powerful radio galaxies with high-ionization nuclear emission;

8. the gas rotation axis and radio axis are aligned to within $10^{\circ}$, but the former is not aligned with the stellar rotation axis.

Acknowledgements. We thank Carlo Stanghellini and Edoardo Trussoni very much for helpful discussions, and Marco Mignoli for providing us useful references. We thank the referee Rita Sambruna for valuable suggestions and comments. E.T. thanks for support the Italian Space Agency (contract ASI-I/088/06/0). G.P. acknowledges ANR and ASI-INAF for support (contracts ANR-06-JC-0047, ASI-INAF I/023/05/0 and ASI-I/088/06/0). S.B. acknowledges financial support from ASI-I/088/06/0. 


\section{References}

Balmaverde, B., Capetti, A., \& Grandi, P. 2006, A\&A, 451, 35

Baum, S. A., \& Heckman, T. M. 1989, ApJ, 336, 681

Baum, S. A., Heckman, T. M., \& van Breugel, W. 1989, ApJ, 389, 208

Belsole, E., Worrall, D. M., \& Hardcastle, M. J. 2006, MNRAS, 366, 339

Bianchi, S., Miniutti, G., Fabian, A. C., et al. 2005, MNRAS, 360, 380

Bianchi, S., Guainazzi, M., \& Chiaberge, M. 2006, A\&A, 448, 499

Brinkman, A. C., Kaastra, J. S., van der Meer, R. L. J., et al. 2002, A\&A, 396, 761

Donato, D., Sambruna, R. M., \& Gliozzi, M. 2004, ApJ, 617, 915

Evans, D. A., Worrall, D. M., Hardcastle, M. J., Kraft, R. P., \& Birkinshaw, M. 2006, ApJ, 642, 96

Fabbiano, G., Miller, L., Trinchieri, G., Longair, M., \& Elvis, M. 1984, ApJ, 277, 115

Fanaroff, B. L., \& Riley, J. A. 1974, MNRAS, 167, 31

Ghisellini, G., Haardt, F., \& Matt, G. 1994, MNRAS, 267, 743

Giovannini, G., Taylor, G. B., Feretti, L., et al. 2005, ApJ, 618, 635

Grandi, P., Malaguti, G., \& Fiocchi, M. 2006, ApJ, 642, 113

Grandi, P., Guainazzi, M., Cappi, M., \& Ponti, G. 2007, MNRAS, 381, L21

Guainazzi, M., \& Bianchi, S. 2007, MNRAS, 374, 1290

Hardcastle, M. J., \& Worrall, D. M. 1999, MNRAS, 309, 969

Hardcastle, M. J., Birkinshaw, M., \& Worrall, D. M. 1998, MNRAS, 294, 615

Hardcastle, M. J., Evans, D. A., \& Croston, J. H. 2006, MNRAS, 370, 1893

Hargrave, P. F., \& McEllin, M. 1975, MNRAS, 173, 37

Heckman, T. M., Carty, T. J., \& Bothun, G. D. 1985, ApJ, 288, 122

der Herder, J., Brinkman, A. C., Kahn, S. M., et al. 2001, A\&A, 365, L7
Jackson, N., \& Rawlings, S. 1997, MNRAS, 286, 241

Kalberla, P. M. W., Burton, W. B., Hartmann, Dap, et al. 2005, A\&A, 440, 775

Kinkhabwala, A., Sako, M., Behar, E., et al. 2002, ApJ, 575, 732

Kirsch, M. 2006, EPIC status of calibration and data analysis,

XMM-SOC-CAL-TN-0018

Koski, A. T. 1978, ApJ, 223, 56

Kraft, R. P., Birkinshaw, M., Hardcastle, M. J., et al. 2007, ApJ, 659, 1008

Laing, R. A., Riley, J. M., \& Longair, M. S. 1983, MNRAS, 204, 151

Leahy, J. P., \& Perley, R. A. 1991, ApJ, 102, 2

Magdziarz, P., \& Zdziarski, A. A. 1995, MNRAS, 273, 837

Miller, C. J., Nichol, R. C., Gómez, P. L., Hopkins, A. M., \& Bernardi, M. 2003, ApJ, 597, 142

Ogle, P. M., Brookings, T., Canizares, C. R., Lee, J. C., \& Marshall, H. L. 2003, A\&A, 402, 849

Piconcelli, E., Bianchi, S., Miniutti, G., et al. 2008, A\&A, 480, 671

Popescu, C. C., Hopp, U., Hagen, H. J., \& Elsaesser, H. 1996, A\&AS, 116, 43

Pounds, K. A., \& Page, K. L. 2005, MNRAS, 360, 1123

Pounds, K. A., \& Vaughan, S. 2006, MNRAS, 368, 707

Risaliti, G. 2002, A\&A, 386, 679

Risaliti, G., Elvis, M., \& Nicastro, F. 2002, ApJ, 571, 234

Sako, M., Kahn, S. M., Paerels, F., et al. 2000a, ApJ, 543, L115

Sako, M., Kahn, S. M., Paerels, F., \& Liedahl, D. A. 2000b, ApJ, 542, 648

Sambruna, R. M., Netzer, H., Kaspi, S., et al. 2001, ApJ, 546, L13S

Sambruna, R., Reeves, J. N., \& Braito, V. 2007, ApJ, 665, 1030

Spergel, D. N., Bean, R., Doré, O., et al. 2007, ApJS, 170, 377

Strüder, L., Briel, U., Dannerl, K., et al. 2001, A\&A, 365, L18

Turner, M. J. L., Abbey, A., Arnaud, M., et al. 2001, A\&A, 365, L27

Urry, M. C., \& Padovani, P. 1995, PASP, 107, 803 